\title{
Terapia Cognitivo-Comportamental para Compras Compulsivas: Um Estudo de Caso Sistemático
}

\author{
Maríndia Brandtner ${ }^{1}$ \\ Escola de Psicologia da IMED \\ Fernanda Barcellos Serralta \\ PPG Psicologia / UNISINOS
}

\begin{abstract}
RESUMO - O presente estudo de caso sistemático examinou o processo e os resultados da terapia cognitivo-comportamental (TCC) de uma compradora compulsiva. A duração do tratamento foi pré-definida em 12 sessões. Todas as sessões foram codificadas com o Psychotherapy Process Q-Set (PQS), um método empírico de avaliação do processo terapêutico. Medidas de resultados incluíram a avaliação de sintomas de depressão, ansiedade e compras compulsivas, bem como do ajustamento social. Houve mudança clinicamente significativa e confiável nos sintomas e no ajustamento social, após o tratamento. A análise do processo indicou que fatores da terapeuta (empatia e responsividade), fatores da paciente (colaboração), fatores da relação (aliança terapêutica) e fatores técnicos (apoio e tarefas de casa) contribuíram para as mudanças observadas.
\end{abstract}

Palavras-chave: comprar compulsivo, transtornos do controle de impulsos, terapia cognitivo-comportamental, processos psicoterapêuticos, psicoterapia

\section{Cognitive-Behavioral Therapy for Compulsive Buying: A Systematic Case Study}

\begin{abstract}
The present systematic case study examined the therapeutic process and results of a cognitive-behavioral therapy (CBT) of a woman with compulsive buyer disorder. The treatment had a fixed duration of 12 sessions. All sessions were rated using the Psychotherapy Process Q-Set (PQS), an empirical method for evaluating the therapeutic process. Outcome measures included assessment of symptoms of depression, anxiety and compulsive buying, and social adjustment. After therapy, there were clinically significant and reliable change in symptoms and social adjustment. The process analysis indicated that therapist's factors (empathy and responsiveness), patient's factors (collaboration), relationship factors (therapeutic alliance) and technical factors (support and homework) contributed to the observed changes.
\end{abstract}

Keywords: compulsive shopping, impulse control disorders, cognitive-behavioral therapy, psychotherapeutic processes, psychotherapy

O comprar compulsivo é um problema psicopatológico associado a fortes e incontroláveis impulsos para comprar (Faber, 2000) e a comportamentos de compras não planejadas, com pouca ou nenhuma avaliação das suas consequências (Grant, Levine, Kim, \& Potenza, 2005; Tavares \& Alarcão, 2008). A síndrome clínica possui características crônicas e repetitivas, com predomínio de preocupações excessivas, desejos e comportamentos envolvendo gastos que levam a consequências adversas e resultam em prejuízo social e psicológico para o indivíduo (Black, 2001; Christenson et al., 1994). A estimativa de sua prevalência na população geral varia de 2 a $8 \%$, podendo estar associada a outros quadros psicopatológicos de eixo I, como transtornos de humor, transtornos de ansiedade e uso abusivo de substâncias (McElroy, Keck, Pope, Smith, \& Strakowski, 1994).

Embora a Terapia Cognitivo-Comportamental (TCC) seja uma abordagem psicoterapêutica de reconhecida validação científica e alinhada ao movimento das práticas baseadas em evidência (Edwards, Dattilio, \& Bromley, 2004; Knapp \& Beck, 2008; Westen, Novotny, \& Thompson-Brenner, 2004), os estudos sobre a efetividade da TCC para o comprar compulsivo são ainda esparsos. Não obstante, há alguma evidência: a TCC em grupo é superior à lista de espera

1 Endereço para correspondências: Rua Alfredo Schuett, 927, Porto Alegre, RS, Brasil. CEP: 91.330-120.E-mail: fernandaserralta@gmail. com
(Mitchell, Burgard, Faber, Crosby, \& Zwaan, 2006; Muller, Arikian, Zwaan, \& Mitchell, 2013) e à ajuda via telefone (Muller et al., 2013) na redução de sintomas de compradores compulsivos.

A TCC inclui procedimentos psicoterapêuticos que combinam a teoria cognitiva, com técnicas derivadas da Análise do Comportamento (Knapp \& Beck, 2008). Conforme esse modelo, diferentes condições psicopatológicas estão associadas a vieses específicos de pensamentos, sentimentos e comportamentos, que influenciam a maneira de uma pessoa incorporar e responder a novas informações (Knapp \& Beck, 2008; Sudak, 2008), sendo que a avaliação cognitiva de estímulos internos ou externos influencia e é influenciada pela ativação de alguns sistemas específicos (Beck \& Dozois, 2011; Longmore \& Worrel, 2007). Sendo assim, a mudança cognitiva gera mudanças no comportamento e vice-versa (Knapp \& Beck, 2008; Sudak, 2008; Wright, Basco, \& Thase, 2008).

A literatura não apresenta estudos que avaliem possíveis mecanismos de ação terapêutica da TCC para compras compulsivas. De modo geral, os estudos em psicoterapia priorizam a avaliação dos seus resultados e ainda pouco se sabe sobre questões fundamentais como quais psicoterapias funcionam para quais pacientes e quais os mecanismos produzem a mudança terapêutica? (Kazdin, 2008; Lemmens et al., 2011). O objetivo do presente estudo é descrever o 
processo de um caso de TCC para o comprar compulsivo, visando estabelecer hipóteses empiricamente sustentadas da mudança observada. Especificamente, o estudo visa verificar os efeitos do tratamento sobre sintomas (comprar compulsivo, ansiedade e depressão) e adequação social, examinar a aproximação da psicoterapia ao seu modelo ideal (protótipo) e verificar possíveis variações no processo, considerando o seu início, meio e fim. Desse modo, busca contribuir, de um lado, para a avaliação da efetividade da TCC para o controle das compras compulsivas, e, de outro, para o acúmulo do conhecimento sobre o processo de mudança em psicoterapias de curta duração.

\section{Método}

\section{Delineamento}

O delineamento proposto é o de Estudo de Caso Sistemático (ECS; Edwards, 1998). O ECS é um delineamento do tipo misto, qualitativo e quantitativo, aplicável a psicoterapias conduzidas em contexto naturalístico (Dattilio, Edwards, \& Fishman, 2010). Busca compreender os fatores que contribuem para a mudança por meio de avaliações repetidas, visando à obtenção de informações válidas, fidedignas e potencialmente replicáveis a outros casos (Kazdin, 2007).

\section{O Caso}

Anna , 34 anos, casada, ensino médio completo, buscou terapia devido à compulsão para compras. $\mathrm{Na}$ ocasião, acumulava dívidas decorrentes dessas compras em diversas lojas, sendo que, somente em uma, o valor do débito era equivalente a 20 vezes seu salário. Fazia uso de antidepressivo há dois anos (150 mg de Venlafaxina/dia) em virtude de sintomas de Episódio Depressivo Maior, conforme avaliação de neurologista. Desde então, não teve outros episódios de humor. Em conformidade com os critérios de inclusão no estudo, Anna apresentava, antes da terapia, sintomas acima do ponto de corte para compras compulsivas, conforme pontuação na Compulsive Buying Scale (CBS; Faber, \& O'Guinn, 1992).

A terapeuta era uma psicóloga especialista em Terapia Cognitivo-Comportamental (TCC) com mais de 20 anos de experiência clínica, que se disponibilizou a colaborar na pesquisa.

O tratamento consistiu em 12 sessões, com frequência semanal de 50 minutos de TCC, realizada em consultório psicológico privado. O número de sessões foi pré-fixado pelas pesquisadoras, tendo como parâmetro a duração de um de tratamento experimental para compradores compulsivos com TCC em grupo que mostrou evidências de eficácia (Mitchell et al., 2006). A terapia não foi manualizada, ou seja, não seguiu procedimentos estandardizados e a terapeuta teve liberdade para escolher como intervir. O plano de tratamento, elaborado pela terapeuta, foi composto de três fases: a fase inicial incluiu a conceitualização cognitiva e a psicoeducação; a fase intermediária privilegiou estratégias de resolução de problemas, intervenções cognitivas e comportamentais, como diálogo socrático, cartões de enfrentamento, identificação de pensamentos permissivos e exposição gradual a estímulos precipitantes da ansiedade; a etapa final do tratamento focalizou estratégias de prevenção de recaída, como a revisão das crenças e comportamentos associados às compras compulsivas e do passo a passo para o enfrentamento das situações de gatilho (Kellett \& Bolton, 2009; Muller \& Mitchell, 2011).

\section{Instrumentos}

Screening para compras compulsivas. Desenvolvida por Faber e O'Guinn (1992), a CBS é utilizada para identificar compradores compulsivos. Foi utilizada a versão em português, desenvolvida por Leite, Rangé, Ribas Junior, Fernandez e Silva (2012).

Avaliação de resultados. Os resultados do tratamento foram avaliados por meio de medidas de autorrelato que avaliaram sintomas de compras compulsivas, depressão e ansiedade, bem como o ajustamento ou a adequação social. Além dessas medidas, foi realizada uma entrevista com a terapeuta, para obter a triangulação de dados, combinando, assim, informações obtidas pelos instrumentos com as observações da terapeuta.

Yale Brown Obsessive Compulsive Scale-Shopping Version (YBOCS - SV). Para acessar cognições e comportamentos relacionados com o comprar compulsivo, foi utilizada a YBOCS-SV (Monohan, Black, \& Gabel, 1996), na sua versão em português do Brasil (Leite, 2011).

Inventário Beck de Depressão (BDI). Para avaliar a intensidade de sintomas de depressão, foi utilizado o BDI (Beck, Steer, \& Brown 1996). Esse inventário foi adaptado para o Brasil por Gorestein, Wang, Argimon e Werlang (2011).

Inventário Beck de Ansiedade (BAI). Para avaliar a intensidade dos sintomas de ansiedade, foi utilizado o BAI (Beck \& Steer, 1990), validado para o Brasil por Cunha (2001).

Escala de Adequação Social (EAS). Para avaliar ajustamento social, foi utilizada a EAS (Weissman, Prusoff, Thompson, Harding, \& Meyers, 1978), na sua versão em português do Brasil (Gorenstein, Andrade, Moreno, Bernick, $\&$ Nicastri, 2000). Esse instrumento mede o funcionamento do respondente em seis áreas: trabalho/escola, social/lazer, relação com a família ampliada, relação conjugal, função parental e funcionamento na família nuclear.

Entrevista. Para complementar a avaliação dos resultados do tratamento, foi realizada uma entrevista semiestruturada com a paciente, realizada após o término da terapia. Nessa entrevista, foi avaliada a percepção da paciente sobre os ganhos obtidos com o tratamento bem como sobre o seu processo de mudança. A entrevista, gravada em áudio, foi realizada pela pesquisadora.

Medida de processo. O processo terapêutico foi avaliado por meio do Psychotherapy Process Q-set (PQS; Jones, 2000). Trata-se de um instrumento do tipo Q-sort, formado por 100 itens que avaliam características do processo de uma psicoterapia, incluindo ações e atitudes do terapeuta, atitudes, 
comportamentos e experiências do paciente, bem como a interação paciente-terapeuta. Os avaliadores examinam a sessão na íntegra, fazem notas de suas impressões e, com o auxílio do manual de instruções do PQS, ordenam os itens em nove pilhas dispostas em um continuum que vai desde os itens menos característicos (categoria 1) até os mais característicos (categoria 9). A escala é ipsativa, isto é, os itens são avaliados uns em relação aos outros. O número de cartões em cada pilha é predeterminado a fim de se obter uma distribuição normal. A versão em português do Brasil do PQS (Serralta et al., 2007), utilizada neste estudo, apresentou coeficientes de fidedignidade comparáveis com os obtidos com o instrumento original (Serralta et al., 2007; Serralta et al., 2010).

\section{Procedimento}

A paciente foi selecionada por conveniência, tendo sido encaminhada à pesquisadora por pessoa familiarizada com o protocolo do presente estudo. A pesquisadora realizou um contato inicial com a participante, explicou os objetivos e procedimentos da pesquisa, obteve o consentimento livre esclarecido e aplicou o instrumento de screening para possível inclusão no estudo. Após a constatação de que a paciente possuía escores superiores ao ponto de corte da CBS (escore de -3,6), a paciente foi encaminhada à terapeuta.

Todas as sessões de tratamento foram gravadas em áudio para posterior transcrição e análise. Os instrumentos de resultado foram aplicados antes da $1^{\text {a }}$ sessão (prétratamento), após a $6^{\mathrm{a}}$ sessão (meio do tratamento), após a $12^{\mathrm{a}}$ sessão (pós-tratamento) e nos seguimentos (follow up 1 e 2) realizados, respectivamente, 16 e 32 semanas após o término do tratamento. No follow-up 1, foi também realizada a entrevista para avaliar ganhos e processo de mudança. Essa entrevista foi gravada em áudio e posteriormente transcrita.

$\mathrm{O}$ instrumento de avaliação de processo (PQS) foi aplicado, por duplas de juízes independentes previamente treinados, às transcrições de todas as sessões de tratamento.

\section{Análise de Dados}

Para avaliar os efeitos do tratamento sobre as medidas de resultados, foi utilizado o Índice de Mudança Confiável (Reliable Change Index, RCI; Jacobson \& Truax, 1991). Esse modelo utiliza critérios clínicos, e não somente estatísticos, para a aferição de resultados de tratamentos (Yoshida, 2008). De acordo com Jacobson e Truax (1991), uma mudança é clinicamente significativa quando o paciente passa de um nível clínico de funcionamento para um não-clínico ou quando a mudança ficou dois desvios-padrão abaixo da média da população clínica (na ausência de dados normativos para populações não-clínicas). O RCI é obtido dividindo-se a diferença entre os escores pós-tratamento e os escores pré-tratamento pelo erro padrão da diferença entre as duas pontuações. Uma pontuação superior a 1,96 no RCI sugere mudanças de maior magnitude do que seria esperado ao acaso.

Para a descrição do processo terapêutico, dois juízes independentes e previamente treinados aplicaram o PQS em todas as sessões de tratamento $(n=12)$. As correlações entre os avaliadores variaram entre $r=0,6$ e $r=0,7$, indicando fidedignidade satisfatória em todas as sessões avaliadas.

Para verificar a adesão da terapia ao protótipo TCC, cada sessão foi correlacionada com o protótipo TCC e também com os protótipos alternativos, psicodinâmico e interpessoal. Como psicoterapias podem apresentar características compatíveis com mais de um modelo ou abordagem, o teste t de Student para amostras pareadas foi realizado para identificar a qual dos modelos o processo aderiu mais significativamente. Todos os protótipos utilizados foram elaborados por Ablon e Jones (1998) a partir de respostas de experts nas referidas abordagens de psicoterapia aos 100 itens do PQS, considerando a sua avaliação da medida da correspondência de cada item a uma sessão ideal da psicoterapia em questão.

As descrições quantitativas do processo terapêutico foram obtidas por meio da média das avaliações dos dois juízes com maior nível de concordância, considerando os 10 itens mais e menos característicos de todo o processo. Para melhor compreender as eventuais diferenças entre as fases do processo terapêutico (inicial, intermediária e final), foi realizada uma ANOVA de medidas repetidas, tendo os itens do PQS como variáveis dependentes. Essa análise foi complementada por análise visual exploratória, realizada por meio da função do diagrama de dispersão para verificar se as variações encontradas poderiam ter significância clínica. Para identificar entre quais fases encontravam-se as diferenças observadas, foi utilizado o teste de Tukey. Todos os procedimentos estatísticos foram realizados por meio do programa SPSS, versão 19.0.

\section{Resultados}

\section{Resultados da Terapia}

No início do tratamento, a paciente apresentava sintomas de depressão em nível moderado, de ansiedade em nível leve, dificuldades em controlar os impulsos referentes às compras e prejuízos importantes no ajustamento social. No término do tratamento, a paciente havia atingido nível de funcionamento não clínico em todas as medidas de resultados. Calculando-se o RCI para os escores da Y-BOCS-SV, foi constatado que já na etapa intermediária do tratamento houve uma redução clinicamente significativa e confiável dos comportamentos de compras compulsivas que levaram a paciente ao tratamento. Essa mudança manteve-se ao final da terapia e nos dois follow-ups realizados, 16 e 32 semanas após o término. Mudanças clinicamente significativas e confiáveis também foram observadas no ajustamento social desde a etapa intermediária, sendo o resultado estável no follow-up 1, mas não no follow-up 2, quando se observou aumento dos escores na EAS, o que indica piora no ajustamento social neste período. Em relação ao pré-tratamento, houve redução dos sintomas de depressão avaliados pelo BDI-II na etapa intermediária, no término, e nos dois follow-ups. No entanto, conforme o RCI, essas alterações foram clinicamente significativas e confiáveis somente no término e no follow- 
up 1. No follow-up 2, o escore do BDI mostrou aumento em relação ao término do follow-up 1. Considerando a ansiedade, em relação ao pré-tratamento, houve diminuição nos escores do BAI na etapa intermediária, no término e no follow-up 2. Conforme o RCI, essas mudanças foram clinicamente significativas e confiáveis. No follow-up 1, o escore no BAI foi elevado, inclusive mais alto do que no pré-tratamento, indicando aumento da ansiedade após o término do tratamento. Os escores da paciente nas medidas de avaliação dos resultados nos diversos períodos podem ser observados na Tabela 1.

A entrevista de seguimento (follow-up 1) mostrou que, além da melhora nos sintomas do comprar compulsivo, a paciente percebeu uma melhora global, incluindo humor e relacionamentos interpessoais. Nessa entrevista, a paciente discorreu sobre as mudanças em alguns pensamentos e crenças disfuncionais obtidas com a terapia: "eu pensava assim: eu sou assim mesmo. Isso aí não é doença! é o meu normal! ... Eu vou sempre ser assim! E ninguém vai me mudar". Revelou que, para não mais comprar o desnecessário, passou a usar estratégias cognitivas para avaliar se realmente precisava do item. Relatou, ainda, ter algum receio de voltar a comprar como antes. Para garantir a manutenção dos ganhos terapêuticos, fazia uso de cartões de enfrentamento (que haviam sido confeccionados durante o tratamento) como um adendo no momento de ir às compras.

Ao ser questionada a que atribuía o resultado do tratamento, a paciente destacou dois aspectos: (a) o relacionamento com a terapeuta - "eu ficava ansiosa prá ir na sessão, prá saber o que a gente ia conversar, tudo, e dai no final ela já me dava o que tinha que fazer durante a semana, ah, era bem bom o nosso relacionamento"; e (b) as tarefas de casa - "eu acho que se não tivesse tido esse teste de eu procurar entrar nos lugares, sabe, porque dai eu saía de lá com aquela ideia... 'eu vou ter que sair agora'... E daí eu pensava assim, 'eu tenho que fazer agora', né, 'não posso deixar passar'... E foi o que ajudou bastante”.

\section{Adesão da Terapia ao Modelo TCC}

A Tabela 2 apresenta as correlações entre o processo das sessões e o processo total (as 12 sessões de terapia) com o protótipo cognitivo-comportamental e com os protótipos

Tabela 1. Medidas de resultados nos periodos de pré-tratamento, intermediário, término e follow up.

\begin{tabular}{lccccc}
\hline Medidas & $\begin{array}{c}\text { Pré- } \\
\text { tratamento }\end{array}$ & Intermediário & Término & $\begin{array}{c}\text { Follow } \\
\text { up 1 }\end{array}$ & $\begin{array}{c}\text { Follow } \\
\text { up 2 }\end{array}$ \\
\hline BDI-II & 25 & 20 & $12^{*}$ & $12^{*}$ & 18 \\
BAI & 10 & $3 *$ & $1 *$ & 22 & $3 *$ \\
EAS & 2.39 & $1.93^{*}$ & $1.74 *$ & $1.91 *$ & 2,26 \\
YBOCS- & 20 & $1 *$ & $0 *$ & $2 *$ & $1 *$ \\
SV & & & & & \\
\hline
\end{tabular}

Nota: A paciente realizou 12 sessões de tratamento. A primeira avaliação de seguimento (follow-up 1) ocorreu 16 após o término do tratamento. A segunda avaliação de seguimento (follow-up 2) ocorreu 32 semanas após o término do tratamento. * Mudança clinicamente significativa e confiável (em relação ao período pré-tratamento), conforme critério de Jacobson e Truax (1991). alternativos, psicodinâmico e interpessoal. Em todas as sessões, foram encontradas correlações significativas com o modelo TCC. A maioria das sessões também mostrou adesão ao modelo interpessoal. Somente a primeira sessão aderiu ao modelo psicodinâmico, sendo que quatro sessões apresentaram processos significativamente opostos a esse modelo. O processo total aderiu significativamente aos modelos TCC e interpessoal. O teste $\mathrm{t}$ de Student para amostras pareadas confirmou que a adesão ao modelo TCC foi significativamente maior $(M=0,620, D P=0,064)$ do que a adesão ao modelo interpessoal $(M=0,249, D P=0,063) ; t(11)=$ $13,261, p=0,000)$.

Tabela 2. Correlações entre processo total e protótipos

\begin{tabular}{|c|c|c|c|}
\hline & $\begin{array}{c}\text { Protótipo } \\
\text { TCC } \\
(\mathbf{N}=100)\end{array}$ & $\begin{array}{c}\text { Protótipo } \\
\text { Interpessoal } \\
(\mathbf{N}=\mathbf{1 0 0})\end{array}$ & $\begin{array}{c}\text { Protótipo } \\
\text { Psicodinâmico } \\
(\mathbf{N}=100)\end{array}$ \\
\hline Sessão 1 & $0,523 * *$ & $0,311 * *$ & $0,338 * *$ \\
\hline Sessão 2 & $0,710^{* *}$ & $0,218^{*}$ & $-0,251^{*}$ \\
\hline Sessão 3 & $0,665^{* *}$ & $0,233^{*}$ & $-0,163$ \\
\hline Sessão 4 & $0,629 * *$ & $0,211^{*}$ & $-0,115$ \\
\hline Sessão 5 & $0,679 * *$ & $0,256^{*}$ & $-0,12$ \\
\hline Sessão 6 & $0,659 * *$ & $0,334 * *$ & 0,065 \\
\hline Sessão 7 & $0,635 * *$ & $0,329 * *$ & $-0,104$ \\
\hline Sessão 8 & $0,552 * *$ & 0,136 & $-0,245^{*}$ \\
\hline Sessão 9 & $0,533 * *$ & $0,322 * *$ & $-0,295 * *$ \\
\hline Sessão 10 & $0,583 * *$ & $0,252^{*}$ & $-0,358 * *$ \\
\hline Sessão 11 & $0,580 * *$ & $0,199 *$ & $-0,187$ \\
\hline Sessão 12 & $0,700 * *$ & 0,19 & $-0,12$ \\
\hline $\begin{array}{c}\text { Processo } \\
\text { Total }\end{array}$ & $0,620 * *$ & $0,249^{*}$ & $-0,129$ \\
\hline
\end{tabular}

\section{Descrição do Processo Terapêutico}

A Tabela 3 mostra os 10 itens do PQS mais e menos característicos do processo terapêutico. A avaliação do processo com o PQS indica que a paciente trazia temas significativos para serem tratados (item 88), contava com a terapeuta para resolver seus problemas (item 52) e, de modo geral, sentia-se ajudada (item 95) e compreendida por ela (item 14). A paciente não tinha dificuldades em compreender comentários da terapeuta (item 5) e tendia a aceitar as suas intervenções (item 42). Durante o processo, a paciente sentia-se segura e confiante (item 44), tinha facilidade em dar início à hora terapêutica (item 25) e exibia uma postura ativa (item 15) e colaborativa (item 87). A terapeuta não era neutra (item 93) e estava afetivamente envolvida e responsiva em relação à paciente (item 9). Sua postura geral era de apoio (item 45). Em suas intervenções, a terapeuta não buscava interpretar ideias, sentimentos ou desejos inconscientes (item 67), exercia ativamente o controle sobre a interação, estruturando a sessão e/ou introduzindo novos assuntos (item 17), solicitando informações (item 31) e dando orientações 
e conselhos explícitos à paciente (item 27). A terapia se concentrou em temas cognitivos, tais como pensamentos e sistema de crenças (item 30), no exame da situação atual ou recente da vida da paciente (item 69) e nas discussões sobre atividades e tarefas específicas para a paciente tentar realizar fora da sessão (item 38).

Com o objetivo de verificar eventuais diferenças nas características do processo terapêutico em suas diferentes etapas, foi realizada uma ANOVA em que as fases da terapia (inicial, intermediária e final) foram as variáveis independentes e os 100 itens do PQS, as variáveis dependentes. Doze variáveis apresentaram diferenças estatisticamente significativas entre as fases do processo terapêutico. Essas variáveis foram, então, submetidas a uma análise visual exploratória, realizada por meio da função diagrama de dispersão, para verificar se as variações encontradas poderiam ter significância clínica. Esse procedimento foi necessário, uma vez que, na escala Q do PQS, as categorias intermediárias $(4,5$ e 6$)$ são categorias neutras ou irrelevantes para a descrição do processo. Sendo assim, na análise visual buscou-se identificar entre esses itens aqueles cuja variação atingia significância clínica (categorias 1, 2 e 3, negativamente saliente; categorias 7, 8, 9 , positivamente saliente). A Tabela 4 apresenta os seis itens que apresentaram diferenças clinicamente relevantes e, ao mesmo tempo, estatisticamente significativas.

Para identificar entre quais fases da terapia encontravamse as diferenças clínicas e estatisticamente significativas encontradas, foi realizado o teste posthoc de Tukey. Os resultados indicaram que a fase intermediária apresentou menos silêncios do que as fases inicial e final (PQS12; $p=0,002)$; a terapeuta mostrou-se mais protetora para com a paciente na fase final da terapia do que nas fases inicial e intermediária (PQS51; $p=0,40)$; na fase inicial do tratamento, a paciente expressava mais reações afetivas abruptas do que nas fases intermediária e final, nas quais $\mathrm{o}$ afeto passou a ser bem modulado (PQS56; $p=0,023$ ); o tema dos relacionamentos interpessoais da paciente foi mais discutido na fase intermediária do que na fase inicial

Tabela 4. Diferenças de médias (ANOVA) entre fases da terapia quanto às características do processo terapêutico

\begin{tabular}{|c|c|c|c|}
\hline Itens do PQS & Gl & $\mathbf{F}$ & Sig. \\
\hline $\begin{array}{l}\text { PQS12 - Ocorrem silêncios durante a } \\
\text { sessão }\end{array}$ & 2 & 16,265 & 0,001 \\
\hline $\begin{array}{l}\text { PQS51- O terapeuta é condescendente } \\
\text { ou protetor para com o paciente }\end{array}$ & 2 & 4,347 & 0,048 \\
\hline $\begin{array}{l}\text { PQS56 - O paciente discute experiências } \\
\text { como se estivesse distante dos seus } \\
\text { sentimentos }\end{array}$ & 2 & 5,820 & 0,024 \\
\hline $\begin{array}{l}\text { PQS63 - Os relacionamentos } \\
\text { interpessoais do paciente são um tema } \\
\text { importante }\end{array}$ & 2 & 7,803 & 0,011 \\
\hline $\begin{array}{l}\text { PQS64 - O amor ou relacionamentos } \\
\text { amorosos são um tópico de discussão }\end{array}$ & 2 & 7,173 & 0,014 \\
\hline PQS87 - O paciente é controlador & 2 & 10,500 & 0,004 \\
\hline
\end{tabular}

Tabela 3. Itens menos e mais característicos do processo terapêtico, conforme PQS

\begin{tabular}{|c|c|c|c|c|}
\hline Item & Conteúdo & Média & Mínima & Máxima \\
\hline \multicolumn{5}{|c|}{ Menos Característicos } \\
\hline 15 & P não inicia assuntos; é passivo. & 1,88 & 1 & 4 \\
\hline 14 & P não se sente entendido pelo terapeuta. & 2,08 & 2 & 3 \\
\hline 44 & P se sente cauteloso ou desconfiado (versus confiante e seguro). & 2,08 & 1 & 4 \\
\hline 42 & P rejeita (versus aceita) os comentários do terapeuta. & 2,25 & 1 & 5 \\
\hline 25 & P tem dificuldade em começar a sessão. & 2,38 & 2 & 4 \\
\hline 87 & P é controlador. & 2,42 & 1 & 5 \\
\hline 67 & $\mathrm{~T}$ interpreta desejos, sentimentos ou idéias inconscientes. & 2,46 & 1 & 5 \\
\hline 5 & P tem dificuldade para compreender o terapeuta. & 2,54 & 1 & 4 \\
\hline 9 & T é distante, indiferente (versus responsivo e envolvido). & 2,58 & 2 & 5 \\
\hline 93 & T é neutro. & 2,67 & 1 & 5 \\
\hline \multicolumn{5}{|c|}{ Mais Característicos } \\
\hline 17 & T exerce controle sobre a interação com o paciente (por exemplo, estruturando novos assuntos). & 7,04 & 5 & 9 \\
\hline 45 & T adota uma atitude de apoio. & 7,04 & 6 & 9 \\
\hline 31 & T solicita mais informação ou elaboração. & 7,08 & 6 & 9 \\
\hline 88 & P traz temas e material significativos. & 7,17 & 6 & 9 \\
\hline 52 & P conta com o terapeuta para resolver seus problemas. & 7,25 & 5 & 9 \\
\hline 95 & P sente-se ajudado. & 7,25 & 6 & 9 \\
\hline 30 & A discussão se concentra em temas cognitivos, isto é, ideias ou sistemas de crenças. & 7,63 & 6 & 9 \\
\hline 27 & T dá orientações e conselhos explícitos. & 7,67 & 5 & 9 \\
\hline 69 & A situação de vida atual ou recente do paciente é enfatizada na discussão. & 7,96 & 7 & 9 \\
\hline 38 & Há discussão sobre atividades ou tarefas específicas para o paciente tentar fazer fora da sessão. & 8,21 & 6 & 9 \\
\hline
\end{tabular}


(PQS63; $p=0,009)$; o relacionamento amoroso da paciente, que não era discutido na fase inicial da terapia, passou a ser um foco da discussão nas sessões intermediárias (PQS64; $p=0,026)$ e finais (PQS64; $p=0,021$ ); a paciente mostrou-se mais colaboradora com a terapeuta na fase intermediária, em comparação com a fase inicial (PQS87; $p=0,003$ ).

\section{Discussão}

Algumas questões originaram este estudo: A terapia funciona? Como a paciente muda? Há variações nos processos que caracterizam as fases inicial, intermediária e final de terapia? Quais os ingredientes ativos do tratamento que podem provocar a mudança? Antes de respondê-las, é importante notar que foi constatado que a terapia estudada pode ser classificada, com segurança, como de orientação cognitivo-comportamental. Embora também apresente elementos que caracterizam outra abordagem de tratamento (no caso, a psicoterapia interpessoal), as correlações são significativamente maiores com o modelo TCC.

O tratamento foi claramente efetivo e, em grande medida, os ganhos terapêuticos foram mantidos ao longo do tempo. Destaca-se que, mesmo após 32 semanas desse breve tratamento, Anna continuava sem sintomas do comprar compulsivo, motivo da busca por tratamento. Na entrevista de follow-up, realizada 16 semanas após a terapia, ficou evidente o quanto a paciente aprendeu sobre o controle dos seus impulsos, até então incontroláveis.

A análise descritiva do processo como um todo, bem como dos elementos mais característicos de cada fase do tratamento, mostrou que uma atitude geral de empatia e responsividade, aliada ao apoio, à diretividade e às tarefas de casa constituíram a maior contribuição da terapeuta para o processo de mudança. Conforme a paciente, o seu bom relacionamento com a terapeuta favoreceu as mudanças positivas que ocorreram durante o tratamento. $\mathrm{O}$ dado da entrevista é corroborado pela descrição quantitativa do processo, obtida por meio do PQS, especialmente quando é examinada a contribuição da paciente e da terapeuta.

A aliança é um fator preditor para bons resultados em psicoterapia, independentemente da linha teórica seguida (Castanguay et al., 1996; Gaston, 1990) e, embora esse fator não seja suficiente para explicar sozinho de que forma os pacientes melhoram (Frieswyck et al., 1986), sabe-se que a percepção do paciente sobre a empatia de seu terapeuta prediz mudanças em psicoterapia (Burns \& Nolen-Hoeksema, 1992). Diversos estudos afirmam que os terapeutas cognitivo-comportamentais são altamente comprometidos em desenvolver e manter uma boa relação terapêutica, sendo vistos como afetivos, carinhosos e competentes por seus pacientes (Keijsers, Schaap, \& Hoodguin, 2000). Esse parece ser o caso da terapeuta de Anna, a qual foi, ao longo da terapia, muito apoiadora e responsiva, além de proporcionar conselho e direção, fazendo com que Anna se sentisse compreendida e motivada para mudar.

Segundo Liese e Franz (2005), o processo de descoberta guiada deve ser apoiador, diretivo e empático, encorajando os pacientes a aprofundar e explorar suas crenças e distorções cognitivas, gerando, assim, novas estratégias de comportamento. Novas estratégias para lidar com o problema foram amplamente estimuladas por meio das tarefas de casa (ver Tabela 3, item 23 do PQS), um dos fatores mais característicos da terapia de Anna. Segundo Liese e Franz, a tarefa de casa é reconhecidamente um fator-chave na melhora de pacientes em TCC. Seu objetivo é proporcionar a prática de habilidades adquiridas e treinadas no setting terapêutico, coletando maiores informações e testando crenças disfuncionais. Há evidências de que realizar tarefas fora da sessão está relacionado a resultados positivos da TCC (Addis \& Jacobson, 2000; Burns \& Nolen-Hoeksema, 1991; Kazantzis, Deane, \& Ronan, 2000; Pearsons, Burns, \& Perloff, 1988). Esse parece ter sido também o caso de Anna, uma vez que as tarefas de casa foram relevantes para seu processo de mudança. Tal como a paciente ressaltou na entrevista de follow-up, as tarefas de casa auxiliaram-na na superação dos sintomas de comprar compulsivo, resgataramlhe a autoconfiança e favoreceram a diminuição da ansiedade frente aos gatilhos.

Sabe-se que não são apenas as técnicas e atitudes dos terapeutas que acionam o processo de mudança numa psicoterapia. Contribuições dos pacientes ao processo são também importantes preditores de mudança. É digna de nota a colaboração de Anna ao longo de todo o tratamento, trazendo para as sessões temas e materiais significativos (ver Tabela 3, item 88 do PQS).

Para Liese e Franz (2005), na TCC, é ideal que o terapeuta explore situações específicas desenvolvidas durante a conceituação de caso. Ao enfatizar a vida atual ou recente da paciente (ver Tabela 3, item 69 do PQS), a terapeuta de Anna a ajudou a examinar ativamente as situações que despertavam a compulsão (gatilhos) para a compra. Durante o tratamento, no entanto, Anna apresentou algumas dificuldades na vida conjugal, as quais também fizeram parte da sessão terapêutica, especialmente na fase intermediária do tratamento. A exploração de temas interpessoais pode explicar a correlação encontrada entre o processo dessa terapia com o protótipo Interpessoal. A exploração dessa temática não implicou perda do foco do tratamento (ver Tabela 3, item 30 do PQS). No entanto, a abordagem mais focada do problema da compra compulsiva na fase inicial do tratamento deu lugar, nas fases intermediária e final, à emergência de problemáticas associadas, de alguma maneira, a ela. A flexibilidade da terapeuta em incorporar e acolher as preocupações da paciente com o seu casamento e com sua filha (ver Tabela 4, item 63 e 64 do PQS) possivelmente também seja um fator que tenha contribuído para o sucesso do tratamento, uma vez que a adesão estrita do terapeuta ao plano de tratamento é relacionada com resultados negativos na TCC (Castanguay et al., 1996). Nesse sentido, é interessante notar que foi nessa etapa que os níveis de colaboração da paciente aumentaram consideravelmente (ver Tabela 4, item 87 do PQS).

A avaliação do processo por meio do PQS e as verbalizações da paciente na entrevista de follow-up mostram que os bons resultados do tratamento são multideterminados. Afirmou Anna: "Não teve assim, 'ah, foi por causa disso'é um conjunto, sabe". Quais foram, então, os elementos que parecem ser mais relevantes para explicar o processo de mudança? 
Sabe-se que, de modo geral, três fatores são essenciais na TCC: (a) o relacionamento colaborativo entre a dupla; (a) a descoberta guiada, que consiste em o terapeuta dirigir o paciente a formular experimentos que serão testados dentro e fora da sessão; (c) a definição conjunta da agenda dos assuntos ou tópicos a serem trabalhados na sessão (Liese \& Franz, 2005; Padesky, 2005). Todos esses fatores interagiram sinergicamente no tratamento de Anna e mostraram-se relevantes para o progresso observado. Não obstante, o caso de Anna sugere que a técnica comportamental (nesse caso, as tarefas de casa e exposições) parece assumir preponderância sobre as demais. A natureza exploratória deste estudo, entretanto, não permite afirmar que esse fator (um dos mais característicos do processo) tenha sido preditor das mudanças observadas e relatadas. Além disso, sabe-se que a frequência da ocorrência de um evento não implica necessariamente em maior impacto ou relevância. Sendo assim, outros estudos são necessários para determinar quais elementos do processo explicam os resultados, por meio de análises de dados mais sofisticadas, como por exemplo, análise de séries temporais e de regressão.

\section{Considerações Finais}

Esse estudo examinou os resultados e o processo de mudança em um estudo de caso sistemático de TCC para compras compulsivas. A investigação sugere que, em casos de compras compulsivas, a TCC é efetiva, não somente promovendo a alteração desse comportamento, mas também melhorando sintomas de depressão e o ajustamento social desses pacientes. Além disso, os resultados indicam que fatores do relacionamento (aliança), da pessoa do terapeuta (empatia e responsividade), do paciente (colaboração) e fatores técnicos (apoio, tarefas de casa) são fundamentais para o progresso do tratamento.

É importante destacar que se trata de um estudo de caso único naturalístico e, portanto, não controlado. A análise de dados foi basicamente descritiva. As limitações metodológicas do presente estudo, aliadas à ausência de pesquisas sobre o processo de mudança na TCC em casos de compras compulsivas, não permitem afirmar em que medida um desses fatores é mais importante que os demais para explicar os resultados do tratamento.

\section{Referências}

Ablon, J. S., \& Jones, E. (1998). How expert clinicans' prototypes of an ideal treatment correlate with outcome in psychodynamic and cognitive-behavioral therapy. Psychology Research, 8(1), 71-83.

Addis, M. E., \& Jacobson, N. S. (2000). A closer look at the treatment rationale and homework compliance in cognitivebehavioral therapy for depression. Cognitive Therapy and Research, 24(3), 313-326.

Beck. A. T., \& Dozois, D. J. A. (2011). Cognitive therapy: Current status and future directions. Annual Review of Medicine, 62, 397-409.
Beck, A. T., \& Steer, R. A. (1990). Manual for the Beck Anxiety Scale. San Antonio, TX: Psychological Corporation.

Beck, A. T., Steer, R. A., \& Brown, G. K. (1996). Manual for the Beck Depression Inventory--ll. San Antonio, TX: Psychological Corporation.

Black, D. (2001). Compulsive buying disorder: Definition, assessment, epidemiology and clinical management. CNS Drugs, 15(1), 17-27.

Burns, D. D., \& Nolen-Hoeksema, S. (1991). Coping styles, homework assignments and the effectiveness of cognitivebehavioral therapy. Journal of Consulting and Clinical Psychology, 59, 305-311.

Burns, D. D., \& Nolen-Hoeksema, S. (1992). Therapeutic empathy and recovery from depression in cognitive-behavioral therapy: A structural equation model. Journal of Consulting and Clinical Psychology, 60, 441-449.

Castonguay, L. G., Goldfried, M. R., Wiser, S., Raue, P., \& Hayes, A. (1996). Predicting the effect of therapy for depression: A study of unique and common factors. Journal of Consulting and Clinical Psychology, 64(3), 497-504.

Christenson, G. A., Faber, R. J., de Zwaan, M., Raymond, N. C., Specker, S. M., Ekern, M. D., Mackenzie, T. B, Crosby, R. D., Crow, S. J., Eckert, E. D., Mussell, M. P., \& Mitchell, J. E. (1994). Compulsive buying: Descriptive characteristics and psychiatric comorbidity. Journal of Clinical Psychiatry, $55,5-11$.

Cunha, J. A. (2001). Escalas Beck. Casa do Psicólogo: São Paulo.

Edwards, D. J. A. (1998). Types of case study work: A conceptual framework for case-based research. Journal of Humanistic Psychology, 38(3), 36-70.

Edwards, D. J. A., Dattilio, F. M., \& Bromley, D. B. (2004). Developing evidence-based practice: The role of case-based research. Professional Psychology: Research and Practice, 35(6), 589-597.

Dattilio, F. M., Edwards, D. J., \& Fishman, D. B. (2010). Case studies within a mixed methods paradigm: Toward a resolution of the alienation between researcher and practitioner in psychotherapy research. Psychotherapy: Theory, Research, Practice, Training, 47(4), 427-441.

Faber, R. J. (2000). A systematic investigation into compulsive buying. In A. L. Benson (Ed.), I shop therefore I am. Compulsive buying and the search for self (pp. 27-54). Northvale, NJ: Aronson.

Faber, R. J., \& O'Guinn, T. C. (1992). A clinical screener for compulsive buying. Journal of Consumer Research, 19, 459469.

Frieswyck, S. H., Allen, J. G., Colson, D. B., Coyne, L., Gabbard, G. O., Horowitz, L., \& Newson, G. (1986). Therapeutic alliance: Its place as a process and outcome variable in dynamic psychotherapy research. Journal of Consulting and Clinical Psychology, 54, 32-38.

Gaston, L. (1990). The concept of the alliance and its role in psychotherapy: Theoretical and empirical considerations. Psychotherapy, 27, 143-153.

Goodman, W. K., Price, L. H., \& Rasmussen, S. A. (1989). The Yale-Brown Obsessive Compulsive Scale: II: Validity. Archives of General Psychology, 46, 1012-1016. 
Gorenstein, C., Andrade, L., Moreno, R. A., Bernick, M., \& Nicastri, S. (2000). Escala de auto-avaliação de adequação social. Validação da versão em língua portuguesa. In C. Gorenstein, L. Andrade, \& A. W. Zuardi (Eds.), Escalas de avaliação clínica em psiquiatria e psicofarmacologia (pp. 401-414). São Paulo: Lemos.

Gorestein, C., Pang, W. Y., Argimon, I. L., \& Werlang, B. G. (2011). Inventário de Depressão de Beck - BDI-II. São Paulo: Casa do Psicólogo.

Grant, J. E., Levine, L., Kim, D., \& Potenza, M. N. (2005). Impulse control disorders in adult psychiatric inpatients. American Journal of Psychiatry, 162(11), 2184-2188.

Jacobson, N. S., \& Truax, P. (1991). Clinical significance: A statistical approach to defining meaningful change in psychotherapy research. Journal of Consulting and Clinical Psychology, 59, 12-19.

Jones, E. E. (2000). Therapeutic action: A guide to psychoanalytic therapy. New Jersey: Aronson.

Kazantzis, N., Deane, F. P., \& Ronan, K. R. (2000). Homework assignments in cognitive and behavioral therapy: A metaanalysis. Clinical Psychology: Science \& Practice, 7, 189-202.

Kazdin, A. E. (2007). Systematic evaluation to improve the quality of patient care: From hope to hopeful. Pragmatic Case Studies in Psychotherapy, 3(4), 37-49.

Kazdin, A. E. (2008, April). Evidence-based treatment and practice. American Psychologist, 63(3), 146-159.

Keijsers, G. P. J., Schaap, C. P. D. R., \& Hoogduin, C. A. L. (2000). The impact of interpersonal patient and therapist behavior on outcome in cognitive-behavioral therapy. Behavior Modification, 24, 264-297.

Kellett, S., \& Bolton, J. V. (2009). Compulsive buying: A cognitivebehavioural model. Clinical Psychology and Psychotherapy, 16, 83-99.

Knapp, P., \& Beck, A. T. (2008). Fundamentos, modelos conceituais, aplicações e pesquisa da terapia cognitiva. Revista Brasileira de Psiquiatria, 30(Supl II), 54-64.

Leite, P. L. (2011). Adaptação transcultural e validação das escalas "The Richmond Compulsive Buying Scale" e "Compulsive Buying Scale" (Unpublished master's thesis). Universidade Federal do Rio de Janeiro, Rio de Janeiro.

Leite, P. L., Rangé, B. P., Ribas Junior, R. C., Fernandez, J. L., \& Silva, A. A. O. (2012). Validação e aferição de fidedignidade da versão brasileira da Compulsive Buying Scale. Revista de Psiquiatria Clínica, 39(3), 100-105.

Lemmens, L. H. J. M., Arntz, A., Peeters, F. P. M. L., Hollon, S. D., Roefs, A., \& Huibers, M. J. H. (2011). Effectiveness, relapse prevention and mechanisms of change of cognitive therapy vs. interpersonal therapy for depression: Study protocol for a randomized controlled trial. Retirado de http://www. trialsjournal.com/content/12/1/150.

Liese, B. S., \& Franz, R. A. (2005). Tratamento dos transtornos por uso de substâncias com a terapia cognitiva: Lições aprendidas e aplicações para o futuro. In P. M. Salkovskis (Ed.), Fronteiras da terapia cognitiva (pp. 407-436). São Paulo: Casa do Psicólogo.

Longmore, R. J., \& Worrell, M. (2007). Do we need to challenge thoughts in cognitive therapy? Clinical Psychology Review, 27, 173-187.
McElroy, S. L., Keck, P. E., Pope, H. G., Smith, J. M., \& Strakowski, S. M. (1994). Compulsive buying: A report of 20 cases. The Journal of Clinical Psychiatry, 55(6), 242-248.

Mitchell, J. A., Burgard, M., Faber, R., Crosby, R. D., \& Zwann, M. (2006). Cognitive behavioral therapy for compulsive buying disorder. Behaviour Research and Therapy, 44, 1859-1865.

Muller, A. A., \& Mitchell, J. E. (Eds.) (2011). Compulsive buying: Clinical foundations and treatment. New York: Routledge.

Muller A, A., Arikian, A., Zwaan, M., \& Mitchell, J. E. (2013). Cognitive-behavioral group therapy versus guided self-help for compulsive buying disorder: A preliminary study. Clinical Psychology and Psychotherapy, 20(1), 28-35.

Monohan, P., Black, D. W., \& Gabel, J. (1996). Reliability and validity of a scale to measure change in persons with compulsive buying. Psychiatry Research, 64, 59-67.

Padesky, C. A. (2005). Desenvolvendo competências do terapeuta cognitivo: Modelos de ensino e supervisão. In P. M. Salkovskis (Ed.), Fronteiras da terapia cognitiva (pp. 236-255). São Paulo: Casa do Psicólogo.

Persons, J. B., Burns, D. D., \& Perloff, J., M. (1988). Predictors of dropout and outcome in cognitive therapy for depression in a private practice setting. Cognitive Therapy and Research, 12, 557-575.

Serralta, F. B., Nunes, M. L. T., \& Eizirik, C. L. (2007). Elaboração da versão em português do Psychotherapy Process Q-Set. Revista de Psiquiatria do Rio Grande do Sul, 29(1), 44-55.

Serralta, F. B., Pole, N., Nunes, M. L. T., Eizirik, C. L., \& Olsen, C. (2010). The process of change in Brief Psychotherapy: Effects of psychodynamic and cognitive-behavioral prototypes. Psychotherapy Research, 20, 564-575.

Sudak, D. M. (2008). Terapia cognitivo-comportamental na prática. Porto Alegre: Artmed.

Tavares, H., \& Alarcão, G. (2008). Psicopatologia da impulsividade. In C. N. Abreu, H. Tavares, \& T. A. Cordás (Eds.), Manual clínico dos transtornos do controle dos impulsos (pp. 19-36). Porto Alegre: Artmed.

Weissman, M., Prusoff, B., Thompson, W., Harding, P., \& Meyers, J. (1978). Social adjustment by self-report in a community sample and in psychiatric outpatients. The Journal of Nervous and Mental Disease, 166(5), 317-326.

Westen, D., Novotny, C. M., \& Thompson-Brenner, H. (2004). The empirical status of empirically supported psychotherapies: Assumptions, findings, and reporting in controlled clinical trials. Psychological Bulletin, 130(4), 631-663.

Wright, J. M., Basco, M. R., \& Thase, M. E. (2008). Aprendendo a terapia cognitivo-comportamental: Um guia ilustrado. Porto Alegre: Artmed.

Yoshida, E. M. P. (2008). Significância clínica de mudança em processo de psicoterapia psicodinâmica breve. Paidéia, 18(40), 305-316.

Young, J. E., Klosko, J. S., \& Weishaar, M. E. (2008). Terapia do Esquema: Guia de técnicas cognitivo-comportamentais inovadoras. Porto Alegre: Artmed.

Recebido em 03.09.2013

Primeira decisão editorial em 23.02.2015

Versão final em 04.04.2015

Aceito em 07.04.2015 